Perspectiva Geográfica

ISSN 0123-3769

Vol.13 • Págs. $127-142$

Diciembre 2008

\title{
Desarrollo y retos de la participación de la sociedad civil en los procesos de Ordenación Territorial*
}

\section{Development and challenges of social society participation in land planning processes.}

\section{María Teresa Rentería Rodríguez**}

\section{Resumen}

La organización de la sociedad civil, como fenómeno global, es relativamente nueva y en los países en vías de desarrollo muy poco ha tenido que ver con los procesos de desarrollo y gobernabilidad; menos aún con los de ordenación territorial, como actor, cuyas decisiones incidieran en esos procesos, pues el Estado había tenido -y sigue teniendouna participación casi exclusiva en la configuración de la realidad política, económica y social, aunque la empresa también ha tenido un peso importante en ello. Este ensayo se centra en la sociedad civil, la cual tradicional mente ha quedado relegada a un plano irrelevante en muchos aspectos que le afectan de modo directo, como es la ordenación del territorio, la educación y la salud, entre otros aspectos. El trabajo se divide en cuatro partes: introducción, génesis y desarrollo de la participación de la sociedad civil, conexión de ésta con los procesos de ordenación territorial y retos de la participación de la sociedad civil.

Palabras clave: desarrollo de la sociedad civil; ordenamiento territorial; organización de la sociedad civil.

Ponencia presentada en el II Si mposi o Col ombo-Mexicano, Uni versidad Pedagógica y Tecnológica de Colombia, 25 al 28 de septiembre de 2007. Tunja, Colombia.

* Profesora e Investigadora del Departamento de Geografía y Ordenación Territorial. Universidad de Guadal aj ara, Méxi co, D. F. E-mail: mayrent r @hot mai l.com 


\section{Abstract}

Civil society organization, as global phenomenon, is relatively new. In developing countries very little of it is accounted for in the governance and development processes; and even less in land planning. In the latter, the State has had, and continues to have, an al most exclusive participation in the configuration of the political, economic and social reality, and not civil society as an actor; though, enterprises also exhibit an important weight in it. This essay centers on civil society, that traditionally has remained relegated to a backstage in many aspects that affect it in a direct way, such as land planning, education and health, among others. The essay is divided in four parts: introduction, genesis and development of the participation of civil society, and its connections and challenges with land planning processes.

Key words: Civil Society Organization; Civil Society Development; land planning. 


\section{Introducción}

El tema de la participación de la sociedad civil, en general, es un tema relevante hoy día. Se podría decir que es un tema que se ha puesto de moda, lanzado a la pasarela, o mejor, a la arena de las contiendas sociales, por los organismos internacionales de más alto nivel. Pero, afortunadamente, no se trata sólo de una cuestión de moda, sino de un tema que tiene que ver con el desarrollo y maduración de nuestras democracias. En los procesos de desarrollo y gobernabilidad, frecuentemente la comunidad se ha quedado a la zaga debido, en buena medida, a una concepción reduccionista de su propia identidad -y la identidad de los otros actores del desarrollo- y de su propia participación en estos procesos. Por su parte, el Estado, en la mayoría de las sociedades en vías de desarrollo -y aún en algunas desarrolladas-, ha tenido poca apertura y sensibilidad frente a las demandas de la sociedad en los diversos campos políticos, económicos y sociales.

En las sociedades democráticas, la gobernabilidad constituye un requisito indispensable para alcanzar un auténtico desarrollo. Pero ni la gobernabilidad ni el desarrollo son tareas que dependan exclusivamente de la gestión políticoadministrativa de nuestros gobiernos; sino más bien, son objetivos que reclaman la colaboración de todos: Estado, empresa y comunidad. En este sentido, la participación ciudadana comienza a ser considerada como uno de los aspectos claves en los procesos de desarrollo local y en la construcción de la gobernabilidad. Cuando la sociedad civil está debidamente organizada constituye un factor relevante en la transformación de la realidad que le circunda y logra mejores condiciones de vida locales y mayores niveles de gobernabilidad. Realiza, además, a través de sus distintas formas de organización, diversos servicios y funciones a los que el Estado no puede llegar, y finalmente, es capaz de incidir eficazmente en la modificación de los marcos legales e institucionales establecidos que favorecen el buen funcionamiento del gobierno, la empresa y la sociedad misma. Indagar, pues, hasta qué punto la sociedad está organizada y es capaz de incidir en los procesos de gobernabilidad y desarrollo, es necesario para encauzar los esfuerzos orientados hacia el fortalecimiento de esos procesos.

\section{Materiales y M étodos}

Las reflexiones vertidas en este artículo tienen su origen en el estudio de algunos documentos básicos utilizados en la Maestría de Gobernabilidad y Desarrollo Humano de la Unversitat Oberta de Catalunya; en la información obtenida en una entrevista a profundidad, realizada a un funcionario público del Ayuntamiento de Zapopan (México) y en la propia experiencia de la autora, en la participación como observadora de diversos talleres de participación ciudadana con motivo de la realización del Plan Ecológico de Ordenamiento Territorial del Municipio de Zapopan. 
Los métodos cualitativos utilizados fueron:

- La observación directa

- La entrevista en profundidad

\section{Génesis y desarrollo de la sociedad civil en A mérica L atina}

En América Latina, la escasa resonancia de las acciones de la sociedad civil se deben, sin duda, a una serie de circunstancias históricas que la han marcado y que están ligadas a su surgimiento y desarrollo. La participación de la sociedad civil es un fenómeno relativamente reciente, localizado temporalmente en la década de los años setenta y ochenta y ha sido el resultado de un proceso que arranca de la época post-independiente, en el siglo XIX, en el que, más que la existencia de una sociedad civil, se encuentra una protosociedad, donde el papel del Estado, e incluso del mercado, ha tenido roles destacados que han contribuido a delinear el perfil de nuestras actuales sociedades. Siguiendo a un autor (Güell 2002), se pueden destacar cinco fases de la participación de la sociedad civil, según se muestra a continuación.

\section{3. $1 \mathrm{~L}$ a etapa post-independiente: 1810 - 1930}

En la primera fase, que va desde la segunda mitad del siglo XIX hasta los años treinta, se puede observar la formación de una sociedad civil incipiente, cuya participación comienza a hacerse manifiesta en los primeros movimientos del artesanado de las ciudades y de los enclaves mineros, inspirados en la literatura anarquista de la época. El Estado asumió el papel de garante del orden público, mediante el establecimiento de un marco institucional y legal que le permitiera corregir los desajustes sociales, para lo cual se valió de la educación dirigida a las masas populares.

El gran logro de esta primera etapa puede cifrarse en la conciencia de que el poder oligárquico no podía ni podría dar cuenta de las demandas de esa nueva sociedad que, no obstante, no llegó a desarrollar una autonomía -aunque sí sus bases-, dadas las relaciones entre el Estado y ésta, que tenía más bien una reacción defensiva frente a aquél. Más tarde, el surgimiento de una clase dirigente ligada a los procesos de fortalecimiento del mercado, llevó a la instauración de una oligarquía que se valió, tanto del populismo como del clientelismo, para su afianzamiento.

\section{2 L a etapa corporativista: $1930-1960$}

En la segunda etapa, delimitada por las crisis de los años treinta hasta los sesenta del siglo XX, la participación de la sociedad se concretó en los movimientos obreros agrupados en torno de los sindicatos. En este contexto, surgió el corporativismo como una forma de hacer efectivos los derechos de los trabajadores. No existía propiamente la sociedad civil, sino una sociedad dependiente de las disposiciones institucionales del Estado, donde sus demandas se resolvían parcialmente por 
la vía corporativista, lo cual dejaba grandes porciones de la población abandonada a un limitado entorno institucional, que no contemplaba los mecanismos de inserción de ésta en los procesos que se seguían en el país, en los distintos entornos políticos, económicos y sociales. Se institucionalizó la participación obrera y se abonaron beneficios materiales a sus afiliados. El Estado adoptó una postura paternalista pero, al mismo tiempo, el reconocimiento derivado de la participación de la sociedad quedó ligado a su conexión con los partidos políticos y más concretamente, con el partido oficial, con la grave limitante de quedar excluidos todos aquellos que no estuvieran asociados a algún sindicato, los cuales, constituían la inmensa mayoría de la población.

Esta alineación con el Estado, fue la principal razón de que no trascendiera la actuación de la sociedad hacia formas auténticas de participación en la construcción del orden social, como tampoco el movimiento social revolucionario logró concretar sus demandas en un avance hacia el reconocimiento de los derechos ciudadanos o humanos.

\subsection{L a etapa de las dictaduras: 1970 - 1980}

El debilitamiento del corporativismo producido por el surgimiento del Estado desarrollista, estuvo acompañado por la intervención de las fuerzas armadas, que interpretaron las movilizaciones sociales como elementos disruptores del orden y que, por lo tanto, había que someter, cuando no suprimir. Así pues, una tercera etapa se puede identificar en el tiempo de las dictaduras. Las contradicciones habidas en la participación de la sociedad civil, debidas en buena parte a las distintas formas de concebir la sociedad, el Estado y el mercado, especialmente las procedentes de los sectores más radicales de la sociedad -los cuales quedaron desmarcados de la participación según el modelo propuesto por el Estado-, abonaron el terreno para el surgimiento de las dictaduras, las cuales identificaron, como problemático, el fenómeno de la participación de la sociedad a través de sus diferentes órganos: sindicatos, partidos políticos progresistas, organizaciones estudiantiles, federaciones campesinas y demás, por lo que, más bien, fueron combatidas o reducidas a su mínima expresión, pero sin llegar a aniquilarlas.

En este ambiente dictatorial y represor, las organizaciones que mejor pudieron pervivir, fueron las organizaciones eclesiales y las ONG, precisamente por carecer del ingrediente político que las podría haber llevado a su desaparición y que sentaron las bases para una participación autónoma posterior de la sociedad civil. De esta forma, surgen las formas más conocidas y difundidas hasta nuestros días: lo que se podría llamar el Tercer Sector. La sociedad civil, por tanto, surgió en un ambiente represivo, aunque en el periodo de las transiciones, fue cuando comenzó a 
tener una incipiente pero más auténtica participación social.

\subsection{L a etapa de la transición hacia las democracias: 1980-1990}

En esta etapa, el Estado ostentaba todavía un gran poder; la sociedad no tenía sino una débil representación y estaba sometida a la estructura institucional, dándose lo que Güell denomina "una externalidad" (Güell, 2002) entre las instituciones y la subjetividad social; es decir, una lejanía entre las pretensiones del Estado y las aspiraciones de la sociedad. En ese ambiente moldeado por un Estado autoritario, la sociedad civil seguía siendo considerada más bien como un elemento desestabilizador por su carácter inorgánico, dentro de los arreglos institucionales de la época de transición. Sin embargo, en general, ésta no dio los resultados de ingobernabilidad que se presuponían, sino que, por el contrario, la situación marcada por la etapa anterior dio como resultado a una sociedad pasiva, que juzgaba como inútil cualquier tipo de participación, -aún la participación política elemental referida a la elección de gobernantes, es decir, a las votaciones-, dificultándose significativamente la formación de una sociedad civil compacta. Sin embargo, no eran del todo inexistentes las manifestaciones de algunas organizaciones de ésta, que empezaban a reflejar, más auténticamente, las preocupaciones de la gente.

El Estado, por su parte, quería rentabilizar aún este incipiente logro social, por lo cual recurrió al reconocimiento y participación de la sociedad, incluyéndola en los foros de consulta del gobierno, con ciertas peculiaridades -contradicciones o precauciones del aparato institucional-, provenientes de la dinámica impuesta por el mismo Estado, como la inclusiónexclusión social en los modos de definir el ordenamiento económico -y aún territorial y social-, así como en sus resultados; la construcción de los sujetos de participación desde el Estado, a través de las convocatorias, que es, a la vez, la forma de reconocerlos y legitimarlos, quedando fuera todos aquellos grupos no alineados a las convocatorias; la multiplicación de las agrupaciones o sujetos sociales que dificulta la generalización de la sociedad civil y que, en la práctica puede, entenderse como la cristalización de las políticas públicas $\mathrm{y}$, finalmente, la fragmentación del espacio público, como resultado de la descentralización de las políticas públicas. Se dio pues, una intermediación institucional entre la participación de la sociedad y el Estado mismo, que puso en riesgo los logros más auténticos de la sociedad civil.

Al final de esta etapa, y no obstante estas contradicciones, se puede valorar como muy positivo el hecho de que la participación se haya institucionalizado y extendido en formas muy variadas, aunque no deje de advertirse al mismo tiempo, como señala Güell, que se haya debilitado su dimensión de construcción y actualización del orden; cuestión que, aunque resulte paradójica, o 
aparentemente contradictoria, es resultado de una menor participación; o, si se quiere, de una participación cualitativa diversa, que responde más al aspecto funcional de la participación que al fundacional.

\subsection{L a etapa de la consolidación de las democracias: 1990}

Al proceso de consolidación de las democracias, corrieron paralelas las medidas económicas de choque, impuestas por los organismos económicos internacionales, como el Banco Mundial y el Fondo Monetario Internacional -el llamado Consenso de Washington- a los países en vías de desarrollo. Los dirigentes de las nacientes democracias, más temerosos del retorno de los regímenes militares, más atentos a los indicadores macroeconómicos y más presionados por el entorno internacional globalizante y por la ofensiva neoimperialista, crearon instituciones que miraban más hacia la solución de los acuciantes problemas macroeconómicos, que hacia la satisfacción de las necesidades y demandas de la sociedad civil en proceso de formación. Si a lo largo de la historia de la existencia de la sociedad civil, ésta ha tenido un peso irrelevante frente al Estado, actualmente, cuando el Estado parece perder entidad, también lo ha tenido frente al mercado, el cual, ahora más que nunca, constituye uno de los poderes fácticos con mayor influencia en el entorno social, incluido el territorio.

Los resultados pueden cifrarse en una retracción de la sociedad civil hacia el ámbito privado, como respuesta al ambiente de indefensión o inseguridad percibida en los ámbitos político y social, reforzándose, de esta manera, las tendencias individualistas que han incidido en el desarrollo de la participación; sobre todo, en el aspecto referido a la deliberación de la construcción del nuevo orden social. Tal situación puede entenderse mejor si se considera el abandono del Estado de su papel integrador de la dinámica social, que dejó la sociedad a merced de la lógica del mercado, ya desde la etapa de los gobiernos dictatoriales y, más tarde, expuesta a los embates de la modernización, en el periodo de la transición.

Sin embargo, es a principios de ésta década cuando el discurso institucional de las grandes organizaciones internacionales, como la ONU -y aún, el mismo Banco Mundial- comienzan a enfatizar la importancia de la participación social en los procesos de desarrollo y gobernabilidad, como elemento clave en los mismos. La adopción de este discurso por parte de los gobiernos, a todos los horizontes, no se hizo esperar; pero, como suele suceder en estos casos, más por razones de maquillaje político que por un auténtico convencimiento, lo cual condujo casi indefectiblemente a un montaje político.

Por otro lado, si bien es cierto todo lo anterior, también lo es el hecho de que la sociedad ha ido organizándose y 
manifestándose cada vez más, en donde son ya no pocos, los ejemplos en los que ésta se agrupa en torno de la consecución de objetivos de diversa índole, entre los que no están ausentes aquellos que tienen una implicación territorial.

\section{Ordenamiento Territorial y participación de la sociedad civil}

Como se ha visto, tradicionalmente la sociedad civil había quedado relegada a un plano irrelevante en muchos aspectos que le afectan de modo directo, como es la ordenación del territorio, la educación y la salud, entre otros. En la actualidad, en América Latina asistimos a un despertar de la sociedad civil, a una mayor conciencia de sí misma y de su potencialidad, si bien es cierto que aún es limitada, pues no se da en todas las sociedades o, por lo menos, no con la misma intensidad, ni con las mismas motivaciones y características, y por supuesto, no con los mismos resultados. Se trata pues, de un fenómeno relativamente reciente y heterogéneo.

\section{1 E I concepto de Ordenamiento Territorial}

Existe un gran número de definiciones de ordenación territorial. Buen ejemplo de ello es el siguiente cuadro resumen (Massiris, 2002): enunciados en la Tabla 1.

\section{Tabla 1. Conceptos de ordenación territorial}

\begin{tabular}{|l|l|}
\hline $\begin{array}{l}\text { Claudius Petit, } \\
\text { especialista francés. } \\
1950\end{array}$ & $\begin{array}{l}\text { "La búsqueda en el ámbito geográfico de la mejor repartición de los hombres } \\
\text { en función de los recursos naturales y de las actividades económicas" (Massé, } \\
\text { 1974, citado por Méndez, 1990:93). }\end{array}$ \\
\hline $\begin{array}{l}\text { G. Saenz de } \\
\text { Buruaga. Espana, } \\
1969\end{array}$ & $\begin{array}{l}\text { "Es el estudio interdisciplinario y prospectivo de la transformación óptima del } \\
\text { espacio regional, y de la distribución de esta formación y de la población total } \\
\text { entre núcleos urbanos con funciones y jerarquías diferentes, con vistas a su } \\
\text { integración en áreas supranacionales" (Pujadas y Font, 1998:11). }\end{array}$ \\
\hline $\begin{array}{l}\text { J. Lajugie y otros. } \\
\text { Francia, 1979 }\end{array}$ & $\begin{array}{l}\text { "El objeto de la ordenación del territorio es de crear, mediante la organización } \\
\text { racional del espacio y por la instalación de equipamientos apropiados, las } \\
\text { condiciones óptimas de valorización de la tierra y los marcos mejor adaptados } \\
\text { al desarrollo humano de los habitantes" (Grenier, 1986). }\end{array}$ \\
\hline $\begin{array}{l}\text { Carta Europea de } \\
\text { Ordenación del } \\
\text { Territorio. 1983 }\end{array}$ & $\begin{array}{l}\text { "Es a la vez una disciplina científica, una técnica administrativa y una política } \\
\text { concebida como un enfoque interdisciplinario y global cuyo objetivo es un } \\
\text { desarrollo equilibrado de las regiones y la organización física del espacio, } \\
\text { según un concepto rector" (CEMAT, 1983). }\end{array}$ \\
\hline $\begin{array}{l}\text { Ley orgánica de } \\
\text { Ordenación del } \\
\text { Territorio. }\end{array}$ & $\begin{array}{l}\text { "Regulación y promoción de la localización de los asentamientos humanos, de } \\
\text { las actividades económicas y sociales de la población, así como el desarrollo } \\
\text { físico espacial, con el fin de lograr una armonía entre el mayor bienestar de la } \\
\text { población, la optimización de la explotación y uso de los recursos naturales y } \\
\text { la protección y valorización del medio ambiente, como objetivos fundamentales } \\
\text { del desarrollo integral" (Congreso de la República de Venezuela, 1983). }\end{array}$ \\
\hline
\end{tabular}




\begin{tabular}{|c|c|}
\hline $\begin{array}{l}\text { Comisión de } \\
\text { Desarrollo y m.a. de } \\
\text { América Latina y el } \\
\text { Caribe. } 1990\end{array}$ & $\begin{array}{l}\text { "Camino que conduce a buscar una distribución geográfica de la población y } \\
\text { sus actividades, de acuerdo con la integridad y potencialidad de los recursos } \\
\text { naturales que conforman el entorno físico y biótico, todo ello en la búsqueda de } \\
\text { unas condiciones de vida mejores" (Comisión de Desarrollo y Medio Ambiente } \\
\text { de América Latina, 1990). }\end{array}$ \\
\hline $\begin{array}{l}\text { Ley de Desarrollo } \\
\text { Territorial. } \\
\text { Colombia, } 1997\end{array}$ & $\begin{array}{l}\text { "Conjunto de acciones político-administrativas y de planificación física } \\
\text { concertadas, emprendidas por los municipios o distritos y áreas metropolitanas..., } \\
\text { para orientar el desarrollo del territorio bajo su jurisdicción y regular la utilización, } \\
\text { transformación y ocupación del espacio, de acuerdo con las estrategias de } \\
\text { desarrollo socioeconómico y en armonía con el medio ambiente y las tradiciones } \\
\text { históricas y culturales" (Congreso de la República de Colombia, 1997). }\end{array}$ \\
\hline $\begin{array}{l}\text { Proyecto de Ley de } \\
\text { OT. Costa Rica, } \\
1998\end{array}$ & $\begin{array}{l}\text { "Proceso dimámico, interactivo e iterativo de diseño de cambios integrales en } \\
\text { las políticas públicas para la clasificación y el uso racional, eficiente y estratégico } \\
\text { del territorio, de acuerdo con criterios económicos, culturales y de capacidad de } \\
\text { carga ecológica y social" (Ministerio de Planificación Nacional, 1998:3). }\end{array}$ \\
\hline $\begin{array}{l}\text { Grupo } \\
\text { Interinstitucional de } \\
\text { OT. México, } 2000\end{array}$ & $\begin{array}{l}\text { "Estrategia de desarrollo socioeconómico que, mediante la adecuada articulación } \\
\text { funcional y espacial de las políticas sectoriales, busca promover patrones } \\
\text { sustentables de ocupación y aprovechamiento de territorio" (SEDESOL y otros, } \\
2000 \text { ). }\end{array}$ \\
\hline $\begin{array}{l}\text { Proyecto de Ley de } \\
\text { Ordenamiento y } \\
\text { Desarrollo } \\
\text { Territorial. Costa } \\
\text { Rica, } 2000 \\
\end{array}$ & $\begin{array}{l}\text { "Conjunto de políticas o directivas expresamente formuladas, normas y } \\
\text { programas que orienten y regulen las actuaciones y procesos de ocupación, } \\
\text { desarrollo y transformación del territorio y el uso del espacio" (Presidencia de } \\
\text { la República de Costa Rica, 2000). }\end{array}$ \\
\hline $\begin{array}{l}\text { Anteproyecto de } \\
\text { Decreto-Ley de } \\
\text { Planificación Física. } \\
\text { Cuba, } 2001\end{array}$ & $\begin{array}{l}\text { "Disciplina técnico administrativa destinada a mejorar los condiciones que tiene } \\
\text { el territorio para las funciones sociales y económicas. Se concreta en los ámbitos } \\
\text { nacional, provincial, municipal y urbano y su contenido fundamental es la } \\
\text { estructuración del espacio físico" (Instituto de Planificación Física, 2001). }\end{array}$ \\
\hline $\begin{array}{l}\text { Proyecto de Ley OT. } \\
\text { Bolivia, } 2001\end{array}$ & $\begin{array}{l}\text { "Proceso de organización del uso y la ocupación del territorio, en función de } \\
\text { sus características biofísicas, ambientales, socioeconómicas, culturales y político- } \\
\text { institucionales, con la finalidad de promover el desarrollo sostenible del país" } \\
\text { (Senado Nacional, 2001). }\end{array}$ \\
\hline
\end{tabular}

Fuente: Massiris, 2002.

\subsection{E I Ordenamiento Territorial y su vinculación con el desarrollo humano}

Como se puede observar en la tabla anterior, la ordenación territorial es concebida como el resultado de la racionalidad de un grupo de expertos que tiene como objeto crear o propiciar mejores condiciones de vida para la población. El Grupo Interinstitucional de Ordenación Territorial mexicano dice, explícitamente, que es una "estrategia de desarrollo socioeconómico" (ver tabla anterior). Se trata, fundamen-talmente, de una política del Estado, como hace notar la Carta Europea de Ordenación del Territorio, a la vez que "una disciplina 
científica y una técnica administrativa" (ver tabla anterior).

En todas estas definiciones, la ordenación territorial aparece, a primera vista, como una externalidad de la sociedad; a ésta, como mera receptora de un efecto que tiene su origen en el Estado, del que recibe su impulso. Sin embargo, al constituir una política del Estado que incide en la organización y ocupación del territorio, se afecta de manera directa la sociedad; por tanto, no puede ser ésta un ente pasivo, sino un actor más en la escena del desarrollo. De esta manera, el desarrollo es concebido cada vez menos, como "algo que nos llega" por la acción del Estado, y más, como una tarea en la que todos: Estado, mercado y sociedad civil, tenemos algo que hacer.

Hoy día, no obstante que la participación de la sociedad civil se considera como un elemento clave, tanto en los procesos de desarrollo local como de ordenación territorial, y de haber obtenido un relativo reconocimiento por parte de las instancias públicas, no recibe todavía la atención necesaria para su inclusión en los mismos. Propuesta desde las más altas esferas internacionales concretamente la ONU a través del Fondo de las Naciones Unidas para el Desarrollo, PNUD- y en general, todas las organizaciones que participan de alguna manera en la Organización Internacional para el Desarrollo, donde se destacan las instituciones financieras como el Banco Mundial o el BID (Kliksberg 1998), la participación de la sociedad civil no tiene un correlato en la agenda pública -concretamente, en aquella que involucra los procesos de ordenación del territorio-.

Massiris afirma que "la ordenación del territorio es un proceso y un instrumento de planificación, de carácter técnicopolítico-administrativo, con el que se pretende configurar, en el largo plazo, una organización del uso y ocupación del territorio, acorde con las potencialidades y limitaciones del mismo, las expectativas y aspiraciones de la población y los objetivos de desarrollo" (Massiris, 2002).

Ahora bien, los objetivos de desarrollo no se conciben actualmente en términos exclusivamente económicos, sino que incluyen metas, las cuales tienen que ver con el desarrollo del ser humano, considerado de forma más integral, donde se plantea el potenciamiento de éste. Desde esta perspectiva el hombre, sin dejar de ser objeto del desarrollo, pasa a ser sujeto de los procesos del desarrollo, lo cual, necesariamente, repercute en la consideración de la sociedad como actor fundamental de éste. Lo importante aquí no es tanto el crecimiento económico, sino el crecimiento de las personas en función de la expansión de sus libertades, así como del surgimiento y fortalecimiento de las matrices y tramas sociales.

Así pues, el desarrollo, concretamente el desarrollo humano, se define como un proceso de ampliación de las capacidades de las personas para elegir

\begin{tabular}{l|l}
\hline 136 & M. Rentería
\end{tabular} 
el tipo de vida que estimen más conveniente. Amartya Sen sostiene que "la capacidad de una persona depende de varios factores, que incluyen las características personales y los arreglos sociales. Por supuesto, una explicación total de la libertad de un individuo debe ir más allá de las capacidades de la vida personal y de prestar atención a los otros objetivos de la persona (por ejemplo metas sociales que no están directamente relacionadas con su propia vida), pero las capacidades humanas constituyen una parte importante de la libertad humana"(Sen, 2004).

En este sentido, la configuración del entorno territorial forma parte de esos "otros objetivos", que van definiendo un estilo de vida y que los miembros de una comunidad tienen derecho a delinear en colaboración con otros agentes, como son el Estado y el mercado. Sen asocia el desarrollo con la ampliación de las libertades que los miembros de una sociedad disfrutan, el cual debería evaluarse en función de estos parámetros (Sen, 2004).

De acuerdo con esto, la libertad que goza la sociedad para definir los espacios que le circundan, es decir para organizar su territorio, estaría contribuyendo de manera muy pobre a elevar los niveles de desarrollo en América Latina, pues aún son muy reducidos los casos en los que la sociedad civil ha tenido un papel importante en la toma de decisiones, en relación con este tópico. Esta manera de concebir el desarrollo implica, necesariamente, arreglos institucionales auténticamente democráticos que, por desgracia, no son todavía muy comunes en la mayoría de nuestras sociedades latinoamericanas, donde la capacidad de acción de la sociedad es aún muy reducida. Por tanto, el objetivo que persigue la participación de los ciudadanos en los asuntos públicos es darle contenido y ampliar la democracia, hacia el avance de lo que se conoce como democracia participativa.

En contraste, cuando en un país sólo existen las libertades básicas -voto cada determinado número de años, libertad de expresión-, se trata de una democracia formal, que puede acabar vacía de contenido. En las sociedades complejas, la participación persigue hacer que los habitantes de un lugar sean más sujetos sociales, con más capacidad para transformar el medio en que viven y de control sobre sus órganos políticos, económicos y administrativos.

\subsection{La participación de la sociedad civil en los procesos de Ordenación Territorial}

Hoy día, no obstante que la participación de la sociedad civil se considera como un elemento clave, tanto en los procesos de desarrollo local, como de ordenación territorial, y de haber obtenido un relativo reconocimiento por parte de las instancias públicas, no recibe todavía la atención necesaria para su inclusión en los mismos. Propuesta desde las más altas esferas internacionales concretamente la ONU a través del Fondo de las Naciones Unidas para el 
Desarrollo, PNUD- y, en general, todas las organizaciones que participan de alguna manera en la Organización Internacional para el Desarrollo, donde se destacan las instituciones financieras como el Banco Mundial o el BID (Kliksberg 1998) la participación de la sociedad civil no tiene un correlato en la agenda pública -concretamente, en aquella que involucra los procesos de ordenación del territorio.

\section{R etos de la participación de la sociedad civil}

Los desafíos que enfrenta la sociedad civil son muchos, de variada índole y como suele suceder en todos los asuntos humanos- de gran complejidad. Sin la pretensión de agotar el tema, se enuncian aquí los que, a parecer de la autora, son los más relevantes, tanto de carácter externo, como interno.

\subsection{Dos percepciones encontradas: el Estado frente a la sociedad civil y la sociedad frente al $E$ stado}

La percepción que se suele tener desde los órganos institucionales del gobierno, en torno de la participación de la sociedad civil, es que resulta ser algo inconsistente, sin organización y carente de normas de educación en los diálogos con sus interlocutores, por lo que los funcionarios públicos se sienten expuestos. Por su parte, la percepción de la sociedad civil en relación con la respuesta del gobierno frente a sus demandas, es que es ignorada; o bien, que es escuchada, pero por pura formalidad, sin un interés real por sus demandas. Ambas visiones corresponden a una parte de la realidad, mayor o menor, según de qué grupo ciudadano se trate, de qué causa y de qué gobierno. Lo cierto es que la participación de la sociedad civil en cualquier proceso, es un fenómeno complejo y, muchas veces, difícil de controlar, por lo que se podría decir que es impredecible.

\subsection{R etos de la participación de la sociedad civil}

Existen muchos obstáculos para que la participación de la sociedad incida efectivamente en los procesos sociales, territoriales o de cualquier otra índole. Además de esa corriente individualista, de la que ya se ha hecho mención, que tiene su raíz en el liberalismo fundamentalmente económico y sobre la cual constituye un verdadero reto su superación, por sus consecuencias en los ámbitos comunitarios y personales; se encuentran otras dificultades que se han separado grosso modo en dificultades ad extra y dificultades ad intra. Los problemas emanados de estos frentes constituyen los retos más importantes que tiene que enfrentar la sociedad para que su participación en los asuntos públicos llegue a incidir eficazmente en su entorno.

\subsection{R etos ad extra de la sociedad civil}

El tema de la participación de la sociedad civil adolece de serias 
dificultades localizadas frecuentemente en la estructura institucional de los gobiernos, los cuales, sin mucha dificultad, suelen moverse al dictado de intereses particulares y no de aquellos que miran al beneficio de la comunidad; o bien, por fuertes intereses del mercado, representados por agentes con importantes relaciones de poder. Una de las razones fundamentales por las que el gobierno no llega a promover una auténtica participación de la sociedad es porque, precisamente, ésta podría llegar a tocar esos intereses.

Las resistencias pueden provenir de actores muy diversos como los partidos políticos, los grupos gremiales, las ONG, funcionarios públicos, etc., y afectan los equilibrios de los grupos de poder; se trata pues, de un proceso altamente político. Por otro lado, los elevados índices de discrecionalidad de los gobiernos, dificultan seriamente los procesos democráticos, pues suelen contravenir no sólo la voluntad expresa de la sociedad, en relación con algunos temas relevantes para ella, sino las mismas disposiciones legales.

En este sentido, uno de los principales retos de la sociedad consiste en exigir e impulsar la implementación de los mecanismos de rendición de cuentas y transparencia de las instituciones públicas, para eliminar o disminuir esos altos niveles de discrecionalidad de las decisiones de los actores del poder público. Otro reto importante, sería abrir mayores y mejores espacios físicos y virtuales para la participación ciudadana y el debate público; sobre todo, abrir espacios en la agenda pública, debido a que no existen los causes legales adecuados para que la participación de la sociedad llegue a concretarse en las decisiones que le conciernen directamente; o bien, si existen, son muy ineficientes o existen solamente en el papel.

Frente al complejo panorama actual, el mayor reto es la elección de un proyecto común que integre a todos los miembros de la sociedad, para lo cual se requiere de espacios, tiempos y lenguajes en los que se pueda deliberar acerca del presente y el futuro que queremos como sociedad. En este sentido, los esfuerzos de ésta, así como los del Estado -y por extraño que pueda parecer, también los del mercado- son imprescindibles en la creación de un sentido de lo social más fuerte, que haga posible ese proyecto, del que dependen no sólo la sociedad, sino el Estado y el mercado mismos.

\subsection{R etos ad intra de la sociedad civil}

Entre las dificultades -y por lo tanto, los retos- que debe afrontar la participación de la sociedad civil al interior de ésta, uno de los principales es la elección de una causa común, suficientemente fuerte que aglutine los esfuerzos, pues muchas veces la ausencia de este elemento es la causa por la cual no se llega a cristalizar la participación; a su vez, la organización de la sociedad constituye otro de los retos importantes, en este sentido.

Es de vital importancia que la definición de los objetivos y la asignación de 
funciones al interior de los grupos organizados estén presentes de manera precisa desde el principio, para evitar desgastes inútiles y dirigir eficazmente las fuerzas hacia la consecución de resultados. En este sentido, la implementación de mecanismos de información, que proporcionen a la sociedad suficientes elementos de juicio para la actuación en un punto determinado, es fundamental, ya que los actores intervienen "desde sus propios marcos de referencia, desde los cuales piensan, definen sus intereses y las estrategias colectivas de acción" (Martinic, 2000).

Pero quizá, más importante aún, es el reto de consolidar el capital social existente, pues éste sensibiliza los miembros de la sociedad, en relación con las necesidades experimentadas por ésta, pues no es infrecuente que, aunque la causa sea de por sí importante, las actitudes individualistas ahoguen las mejores causas sociales. El impulso y fortalecimiento del capital social mediante el fomento de los valores de alto contenido social, como el cooperativismo, la solidaridad, la confianza y otros por la misma línea, constituye uno de los más importantes retos al interior de la sociedad que, necesariamente, aflora en la participación ciudadana.

La existencia de un capital social robusto también propicia intercambios firmes pero a la vez amables- con el resto de los actores y posibilita el intercambio de opiniones en un clima de respeto y confianza que conduce al diálogo y propicia los consensos. Finalmente, vale la pena mencionar que uno de los retos más difíciles -quizá el más difícil- es la prueba del tiempo: la resistencia en la lucha que se sostiene a lo largo de las semanas, de los meses y aún de los años, produce un desgaste en los actores sociales que muchas veces termina venciéndolos. El sentido de responsabilidad frente a las demandas sociales -contenido también en el capital social- es uno de los valores más importantes de nuestro tiempo y uno de los mayores retos.

\section{Conclusiones}

Las innegables dificultades que afronta la sociedad civil, desde dentro como desde fuera de ella, su pasado histórico y su relativamente reciente aparición, son cuestiones que influyen en su escasa incidencia en los campos político, social, económico y, desde luego, territorial. Sin embargo, la sociedad civil está llamada a redescubrirse a sí misma y a redescubrir su propio potencial en la construcción de su entorno -político, económico y social-, el cual, necesariamente, tiene un referente territorial, que debe estar de acuerdo con sus aspiraciones. Muestra de que la sociedad civil va dando pasos en este sentido es su cada vez más frecuente participación en los asuntos públicos que más de alguna vez ha sido exitosa-, al tiempo que alienta la esperanza de una más amplia, madura y eficaz participación. 


\section{Literatura citada}

Güell, P. 2002. "Participación, capital y cohesión social. La construcción social de la gobernabilidad: capital social, participación y cultura cívica. (Una perspectiva desde América Latina)", Documento base, módulo 5 "Participación" de la Maestría en Gobernabilidad y Desarrollo Humano de la Universitat Oberta de Catalunya (UOC).

Kliksberg, B. 1998. "Seis tesis no convencionales sobre participación”, en Instituciones y Desarrollo, Diciembre.

http://www.participar.org/documentos/Seis_Tesis_Klisberg.pdf. Se tuvo acceso en noviembre de 2008

Martinic, S. 2000. "La construcción social de las reformas educativas y de salud en América Latina”, Reforma y Democracia. No. 18, Octubre, Caracas.

Massiris, Á. 2002. “Ordenación del territorio en América Latina, Scripta Nova 2000, Revista electrónica de ciencias sociales de la Universidad de Barcelona, Vol. VI, núm. 125, 1 de octubre.

http://www.ub.es/geocrit/sn/sn-125.htm. Se tuvo acceso en marzo de 2009

Sen, A. 2004. "Capacidad y bienestar” en Martha C. Nussbaum y Amartya Sen (Comp.): La calidad de vida, pp. 95-100.4 ${ }^{\mathrm{a}}$ reimpresión, México, D.F.: Fondo de Cultura Económica.

Fecha de recepción: 16 de junio de 2008

Fecha de aprobación: 17 de diciembre de 2008 
